Revista Perspectivas Online: Exatas \& Engenharias Dezembro de 2018, Vol.8, n 23, p. 18 - 27

ISSN: 2236-885X (Online)

DOI: $10.25242 / 885 X 82320181685$

\title{
ESTUDO DA RIGIDEZ DOS SUPORTES DA BANCADA DE ANÁLISE DE VIBRAÇÕES
}

\author{
João Marcelo Cardoso Carvalho', Cláudia Márcia R. Machado Albernaz ${ }^{12}$, Diego Soares \\ Azevedo ${ }^{1}$
}

\section{RESUMO}

ALBERNAZ, C, M, R, M, CARVAlHO, J; AZEVEDO, D, S. Análise da Rigidez dos Suportes de uma Bancada de Análise de Vibrações. Perspectivas Online: Exatas \& Engenharias, v. 8, n.23, p.18-27,2018.

Com base na necessidade de verificar a rigidez de um conjunto de suportes, confeccionados com o propósito de sustentar os mancais e rolamentos de uma bancada didática de análise de vibrações, esse artigo propõe uma abordagem teórica do problema. No primeiro momento foram apresentadas as equações que regem o movimento vibratório de uma estrutura sob a ação de uma força desbalanceadora, a maneira como essas equações seriam usadas para solucionar o problema e os softwares de análise utilizados na análise. Em seguida foram realizados os cálculos conforme as equações de movimento e os resultados obtidos durante a análise computacional no software Ansys. Então os resultados encontrados foram discutidos e a real necessidade de serem feitas modificações ou não na estrutura dos suportes. Foi observado que a adição das mãos-francesas, sob uma primeira perspectiva teórica, foi desnecessária. Contudo, dentro de um escopo realista fica em aberto a necessidade dessas estruturas para a bancada.

Palavras-chave: Vibrações Mecânicas; Desbalanceamento Rotativo; Simulação Estrutural. 


\begin{abstract}
Based on the need to verify the stiffness of a set of supports, made to hold the bearings of a didactic bench for analysis of vibrations, this article proposes a theoretical approach to the problem. First, the equations that govern the vibratory movement of a structure under the action of an unbalance force, the way in which these equations would be used to solve the problem and the analysis software used in the parsing were presented. Then the

the equations of motion and the results obtained during the computational analysis in Ansys software. Finally, the results found and the real need to make modifications or not in the structure of the supports were discussed. It was observed that the addition of the "French hands", from a first theoretical perspective, were unnecessary. However, within a realistic scope the need for these structures for the workbench remains open.
\end{abstract} calculations were performed according to

Keywords: Mechanical Vibrations; Rotary Imbalance; Structural Simulation.

\footnotetext{
${ }^{1}$ Institutos Superiores de Ensino do CENSA - ISECENSA - Rua Salvador Correa, 139, Centro, Campos dos Goytacazes, RJ, CEP: 28035-310, Brasil;

${ }^{2}$ Universidade Estadual do Norte Fluminense Darcy Ribeiro - UENF - Av. Alberto Lamego, 2000, Parque Califórnia, Campos dos Goytacazes, RJ, CEP: 28013-602, Brasil.

(*) e-mail: cmrmachado@gmail.com

Data de recebimento: 10/07/18. Aceito para publicação: 20/12/18.
} 


\section{INTRODUÇÃO}

O estudo de vibrações é fundamental quando se planeja projetar um equipamento ou avaliar o comportamento de uma estrutura já construída. Vibração pode ser qualquer movimento que sofra uma repetição passado certo tempo e a teoria de vibração trata de estudar essas oscilações e como as forças associadas a ela ser comportam (RAO, 2010).

Um sistema está sob uma condição de vibração forçada quando uma energia externa é introduzida no sistema de vibração, normalmente por meio de uma força aplicada ou uma excitação do deslocamento imposta (BALACHANDRAN; MAGRAB, 2008).

Quando essa força de excitação ocorre de maneira periódica diz-se que ela é harmônica. Esse tipo de excitação é justamente o resultado de um sistema rotativo com uma massa desbalanceada. Essa excitação é transmitida para todos os outros elementos, tais como eixos, suportes, mancais e etc.

Segundo Sousa (2005), desbalanceamento pode ser definido como a existência de uma massa que se posiciona excentricamente em relação ao eixo de rotação de um rotor e desloca o seu centro de gravidade perpendicularmente.

De acordo com Lima (2014), o desbalanceamento pode estar ligado a diversas causas como assimetrias, deformações, imperfeições na matéria prima, problemas na montagem das maquinas e é uma das principais fontes de vibração.

Durante a construção de uma bancada didática de análise de vibrações para os laboratórios do ISECENSA, ao realizar o processo de fresagem em um suporte que tem como função sustentar os mancais, foi observado um comportamento peculiar, quando a estrutura foi submetida à força de corte apresentou uma trepidação muito elevada, levando a acreditar que não possuiria rigidez suficiente para suportar as forças de desbalanceamento durante os ensaios de vibração.

O objetivo desse artigo é estudar o comportamento desse modelo de suporte de mancal, sob a ação dessa excitação harmônica causada por uma massa desbalanceada e justificar ou não a adição de um conjunto de mãos-francesas a sua estrutura, as quais foram adicionadas empiricamente como uma possível solução, adotada durante a construção da bancada.

A avaliação da estrutura do suporte será realizada primeiramente usando os cálculos de vibração mostrados na literatura e em seguida analisados computacionalmente utilizando o software de elementos finitos Ansys.

\section{METODOLOGIA}

A bancada em questão, mostrada na Figura 1, possui um motor sustentado por um suporte, ligado ao eixo rotativo por um acoplamento elástico e dois suportes para sustentação de dois mancais de deslizamento além de um disco para realizar o balanceamento, que será o responsável por criar a força de excitação periódica.

Persp. Online: exatas \& eng., Campos dos Goytacazes, 23 (08) 18-27 - 2018

seer.perspectivasonline.com.br 


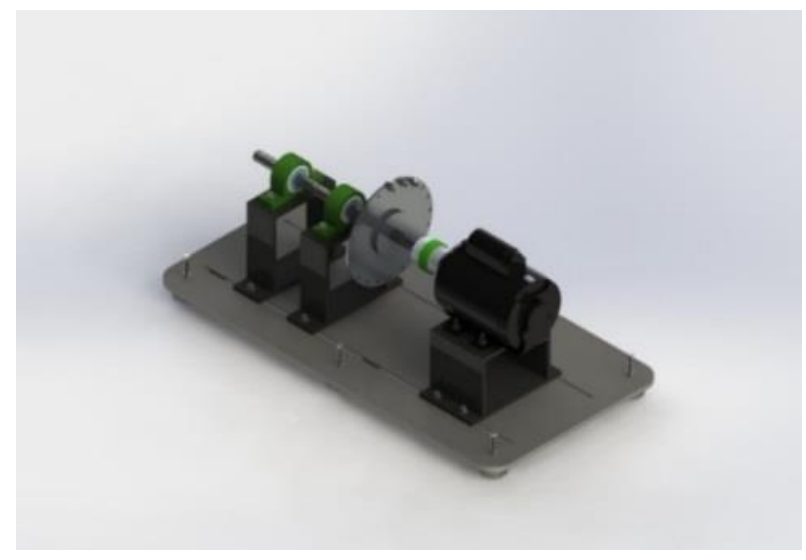

Figura 1 - Modelo CAD da Bancada.

Primeiramente foi calculada a rigidez equivalente dos suportes e suas respectivas massas. Em seguida usando a equação 1 foi calculada a frequência natural da estrutura. Com esses valores pode-se ter uma primeira impressão sobre a rigidez da estrutura.

$$
\omega_{n}=\sqrt{\frac{k}{M}}
$$

Onde $k$ é a rigidez equivalente da estrutura em N/m e $M$ é a sua massa total em $\mathrm{kg}$. $\mathrm{O}$ valor da frequencia natural é dado em rad/s.

Para calcular a rigidez equivalente de estruturas como vigas bi-apoiadas ou sob a ação de uma força axial, como mostrado na figura 2, utiliza-se as equações 2 e 3 respectivamente. $O$ momento de inércia é representado pela letra $I$, o módulo de elasticidade do material pela letra $E$, a área da secção transversal por $A$ e as dimensões da distância de uma carga pontual até os suportes onde a viga está engastada por $a$ e $b$.

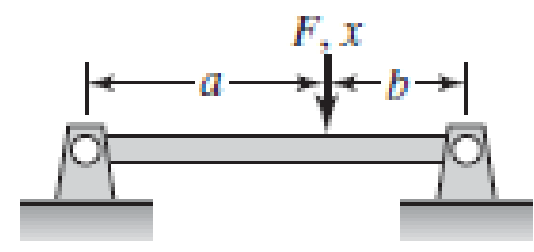

Figura 2 - Representação da força que atua sobre uma barra bi-apoiada.

Fonte: Balachandran, 2011.

$$
\begin{gathered}
k=\frac{A E}{L} \\
k=\frac{3 E I(a+b)}{(a b)^{2}}
\end{gathered}
$$


Como passo seguinte, foram calculadas as forças de reação que esses suportes sofrem quando a máquina está perfeitamente balanceada. As forças presente nesse caso são apenas o peso dos mancais, com o rolamento, e o peso do disco de desbalanceamento. Aplica-se então a teoria do equilíbrio estático, equações 4 e 5 respectivamente, em conjunto com o software Ftools para facilitar os cálculos.

$$
\begin{aligned}
& \sum F y=0 \\
& \sum M=0
\end{aligned}
$$

A resposta de um sistema amortecido excitado harmonicamente pode ser descrito pela equação 6, onde $m$ é a massa do sistema em $\mathrm{kg}, c$ é o coeficiente de amortecimento em $\mathrm{Kg} / \mathrm{s}$, $k$ é a rigidez do elemento mola em $\mathrm{N} / \mathrm{m}$ e $F_{o}$ é a amplitude máxima da força de excitação.

$$
m \ddot{x}+c \dot{x}+k x=f(t)
$$

Quando desbalanceado, um rotor ou eixo passa a sofrer uma força de excitação harmônica, que é transmitida para os mancais, e consequentemente para o topo dos suportes. Como essa força tem um comportamento harmônico, a equação 7 é capaz de descrever o seu comportamento em função do tempo e a Figura 2 ilustra como o movimento ocorre.

$$
f(t)=m_{o} \varepsilon \omega^{2} \operatorname{sen}(\omega t)
$$

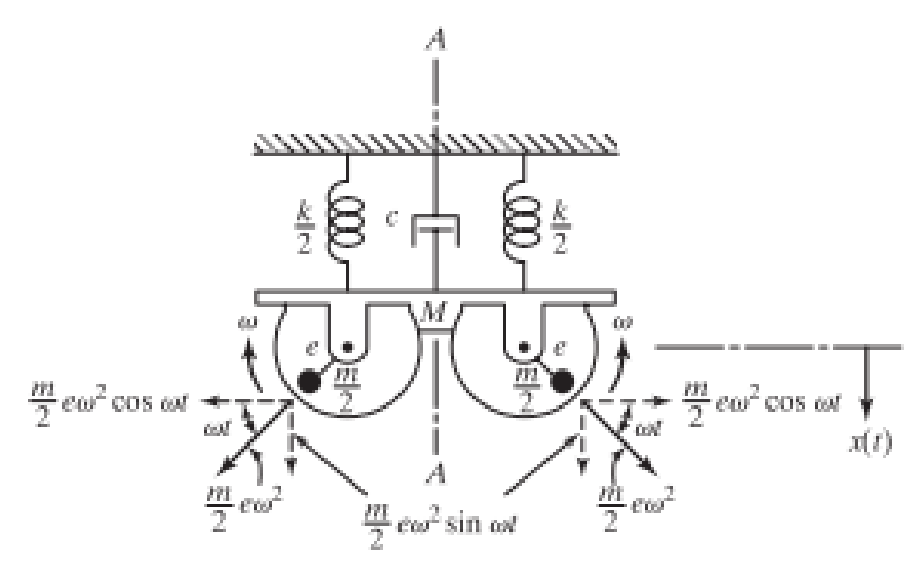

Figura 3 - Comportamento de uma massa desbalanceada. Fonte: Rao, 2008.

Onde $m_{o}$ é a massa desbalanceada em $\mathrm{kg}, m$ é a massa total do disco de balanceamento em $\mathrm{kg}, \varepsilon$ é a distância da massa até o centro geométrico do corpo e $\omega$ é a frequência de excitação $\mathrm{em} \mathrm{rad} / \mathrm{s}$ (velocidade de rotação do disco).

A resposta a excitação em termos de deslocamento vai possuir também um comportamento harmônico, descrito pela equação 8. Resolvendo a equação 6 com as equações 7 e 8 obtém-se a equação 9. 


$$
\begin{gathered}
x(t)=X \operatorname{sen}(\omega t-\varphi) \\
\frac{M X}{m \theta}=\frac{r^{2}}{\sqrt{(1-r)^{2}+(2 \zeta r)^{2}}}
\end{gathered}
$$

Onde $\varphi$ é o angulo de fase de vibração, $X$ é a amplitude de vibração, $r=\omega / \omega_{n}$ é a razão entre as frequências e $\zeta=c / \sqrt{\mathrm{km}}$ é a razão de amortecimento.

Para calcular a parcela de força que será transmitida para o mancal $f_{s}$, utiliza-se a equação 10, onde $a$ é a distância em que o centro geométrico do disco está para mancal, e para calcular o deslocamento máximo $Y$ sofrido pelo suporte onde o mancal está apoiado, com um amortecimento desprezível, utiliza-se a equação 11.

$$
\begin{aligned}
& f_{s}=\frac{a}{l} m_{o} \varepsilon \omega^{2} \\
& Y=\frac{f_{s}}{\left(k-m \omega^{2}\right)}
\end{aligned}
$$

Outro parâmetro que foi utilizado para verificar a rigidez da estrutura foram os modos de vibração em cada um dos modelos, a partir de uma análise modal, utilizando o software Ansys. Primeiro foi feira uma análise de vibração livre-livre e em seguida uma análise em vibração apoiada. Para esta análise o modelo CAD foi simplificado utilizando o software SpaceClaim, conforme mostrado na figura 3, afim de facilitar a geração da malha.

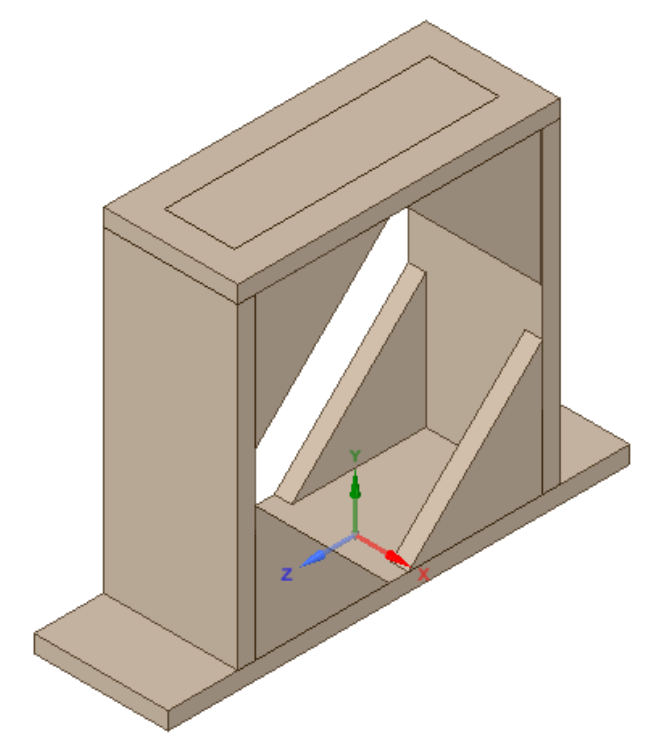

Figura 4 - Modelo Simplificado do Suporte com Mão-Francesa. 


\section{RESULTADOS}

Dividindo a estrutura em 4 partes estruturais, eixo, suporte A, suporte B e suporte motor, e aplicando as equações 2 e 3 , de rigidez equivalentes, os resultados obtidos são representados na tabela 1 , junto com suas massas equivalentes e a frequência natural de cada uma dessas estruturas. $\mathrm{O}$ eixo foi dividido em mais 3 partes devido a diferença de diâmetros e a presença dos apoios. A figura 4 mostra como esse eixo foi dividido.

Tabela 1 - Rigidez Equivalente das Estruturas da Bancada.

\begin{tabular}{cccc}
\hline Estrutura & $\begin{array}{c}\text { Rigidez Equivalente } \\
(\mathbf{k N / m})\end{array}$ & $\begin{array}{c}\text { Freq. } \\
\text { natural } \\
(\mathbf{R a d} / \mathbf{s})\end{array}$ & Massa $(\mathbf{K g})$ \\
\hline $\begin{array}{c}\text { Suporte A e } \\
\text { B }\end{array}$ & 9915,2 & 1726,3 & 3,327 \\
Suporte & 15731,9 & 1661,8 & 5,697 \\
Motor & & & \\
Eixo A & $2,613 \mathrm{E}+6$ & 156378,63 & 0,107 \\
Eixo B & 91616 & 8569,5 & 1,248 \\
Eixo C & $2,99 \mathrm{E}+8$ & 15341,83 & 1,270 \\
\hline
\end{tabular}

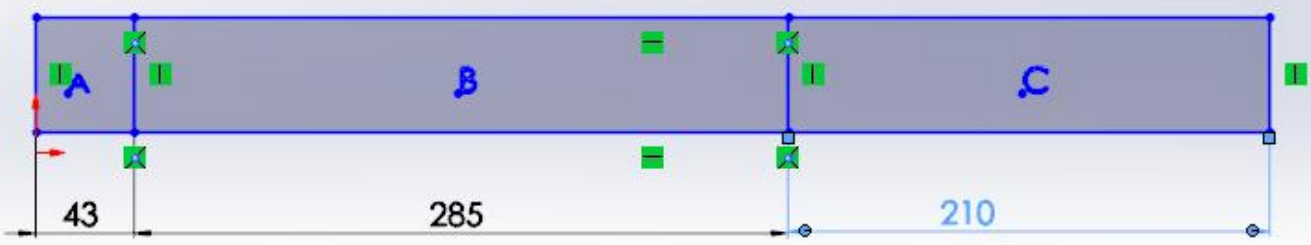

Figura 2 - Dimensões das divisões do eixo.

O motor utilizado para girar o eixo possui uma rotação de $350 \mathrm{rad} / \mathrm{s}$, a massa do parafuso (massa desbalanceadora) é de 12 gramas, e sua distância do eixo é de 110 milímetros. Aplicando então a equação 5 com essas informações obtém-se a intensidade da força desbalanceadora:

$$
f(t)=0,020 \times 0,11 \times(350)^{2} \times \operatorname{sen}(350 t)=269,5 \operatorname{sen}(350 t) N
$$

A deflexão máxima causada pela força desbalanceadora nessas condições é calculada quando a amplitude é máxima. Dessa forma, utilizando a equação 7 e considerando um amortecimento desprezível, descobre-se que:

$$
X=\frac{161,7}{\left(3 \times 10^{8}-1,270 \times 350^{2}\right)}=5,396 \times 10^{-4} \mathrm{~mm}
$$


Levando em conta que o disco de balanceamento se encontra exatamente na metade da distância entre o mancal $\mathrm{B}$ e o motor, a força transmitida ao mancal será igual à metade da força máxima causada pelo desbalanceamento se aplica a equação 8. Assim:

$$
f_{s}=0,5 \times 161,7=80,85 \mathrm{~N}
$$

Aplicando os resultados até aqui encontrados na equação 9, obtém-se os seguintes resultados para o deslocamento máximo do suporte se forem utilizados a sua massa e rigidez equivalente:

$$
Y_{\text {mancal }}=\frac{80,85}{\left(9,92 \times 10^{6}-3,327 \times 350^{2}\right)}=8,499 \times 10^{-3} \mathrm{~mm}
$$

Para o suporte do motor são realizados cálculos semelhantes. Como o motor encontra a mesma distância que o mancal B se encontra do disco, tem-se que a força transmitida será a mesma. O deslocamento transmitido será calculado da mesma maneira como na equação 7 , porém usando os parâmetros corretos de massa e rigidez equivalente. O que resulta em:

$$
Y_{\text {motor }}=\frac{80,85}{\left(1,573 \times 10^{7}-5,697 \times 350^{2}\right)}=5,378 \times 10^{-3} \mathrm{~mm}
$$

Quanto à estrutura dos suportes dos mancais e para o suporte do motor com mãosfrancesas, obtém-se os seguintes resultados com o software Ansys e seu módulo Mechanical para os modos de frequências naturais na Tabela 2.

Tabela 2 - Modos de vibração do suporte dos mancais e do motor com mãos-francesas.

\begin{tabular}{ccc}
\hline Modo & $\begin{array}{c}\text { Frequências Naturais dos } \\
\text { Suportes do Mancal (Hz) }\end{array}$ & $\begin{array}{c}\text { Frequências Naturais } \\
\text { do Suporte do Motor } \\
(\mathbf{H z})\end{array}$ \\
\hline 1 & 558,48 & 399,02 \\
2 & 644,46 & 512,25 \\
3 & 1280,80 & 755,49 \\
4 & 1478,60 & 2402,4 \\
5 & 1848,10 & 2434,5 \\
6 & 2437,10 & 2507,9 \\
\hline
\end{tabular}

\section{DISCUSSÃO}

Observa-se-que com os resultados apresentados, para o desbalanceamento com 1 grau de liberdade e para apenas uma massa de desbalanceamento, um deslocamento muito pequeno na ordem de milésimos de milímetros. Tal nível de vibração, segundo o que é mostrado no trabalho de Sousa (2005), é quase que inofensivo para um sistema que esteja devidamente fixado, com parafusos com o torque correto e com o eixo devidamente alinhado. 
Observa-se também que as frequências naturais da estrutura com ou sem o conjunto de mãos-francesas, são bem altos, como mostrado na tabela 2 , e que a velocidade de rotação do motor é bem menor que essas frequências naturais. Lima (2015) mostra em seu trabalho que a frequência modal mais importante seria a que mais se aproxima da velocidade de rotação do motor elétrico. Contudo nesse caso, nenhuma delas se aproxima o suficiente para gerar qualquer tipo de preocupação.

Acredita-se que o que pode ter acontecido durante o trabalho de fresagem das superfícies dos suportes, tanto do motor quanto dos mancais, foi que a força de corte da ferramenta tenha sido excessiva para o trabalho em questão.

Outra hipótese seria uma velocidade de avanço muito alta ou uma profundidade de corte acima do necessário possa ter causado o aumento da força. Além disso, a ferramenta utilizada não era nova, o que poderia implicar que alguns de seus dentes não estavam afiados o suficiente, causando uma periodicidade na força de corte que pode ter amplificado o deslocamento da massa.

\section{CONCLUSÕES}

O trabalho realizado nos permite concluir com base nos resultados dos cálculos que para um sistema com um grau de liberdade com apenas uma massa desbalanceadora não é necessário o uso do conjunto de mãos-francesas. Fica claro, portanto, que a estrutura já possuía rigidez equivalente para suportar as vibrações causadas pelas massas desbalanceadoras.

Todavia, é de suma importância destacar que existem perguntas geradas na discussão que precisam ser respondidas, tais como o torque dos parafusos que prenderam os suportes na fresa, os parâmetros de corte da ferramenta utilizada.

O nível de vibração que o processo de fresagem gerou nas estruturas não pode ser ignorado, um estudo mais aprofundado poderá ser realizado em trabalhos futuros nos quais o foco poderá ser as possíveis fontes de vibração causadas pela ferramenta de corte. Outra ideia que pode ser examinada mais a fundo seria a medição dos níveis de vibração em diversas profundidades de corte e velocidades de avanço com o uso de dispositivos como acelerômetros.

Esse trabalho atingiu seu objetivo ao estudar o comportamento do modelo de suporte de mancal objeto do estudo, sob a ação de uma excitação harmônica causada por uma massa desbalanceada, podendo justificar baseado em parâmetros e resultados obtidos a não necessidade do reforço da estrutura.

\section{REFERÊNCIAS}

BALACHANDRAN, Balakumar; MAGRAB, Edward B. Vibrações mecânicas. São Paulo: Cengage Learning, 2011.

LIMA, Israel Antônio Macedo de. Proposição de uma bancada didática para análise de vibração em manutenção preditiva. 2015.

RAO, Singiresu S. Vibrações mecânicas. Pearson Educación, 2009.

SOUSA, Walter dos Santos. Desenvolvimento de um Sistema Aplicativo em Labview para o

Persp. Online: exatas \& eng., Campos dos Goytacazes, 23 (08) 18-27 - 2018

seer.perspectivasonline.com.br 
Monitoramento de Máquinas Rotativas com um Módulo de Balanceamento de Rotores. Trabalho de Conclusão de Curso pela UFPA, 2005. 